\section{Giant Diverticulum of the Ascending Colon}

J. C. Kang, S. W. Jao, P. C. Chao, C. C. Wu

Division of Colorectal Surgery, Department of Surgery, Tri-Service General Hospital, National Defense Medical Center, Taipei, Taiwan, R.O.C.

\section{Corresponding Author}

S. W. Jao, M.D.

Division of Colorectal Surgery

Tri-Service General Hospital

FL4, No. 38-4, Section 3

Ting Chow Road

Taipei, R.O.C.

Taiwan

Fax: $\quad+886-2-23681840$

E-mail: jckang@ms22.hinet.net

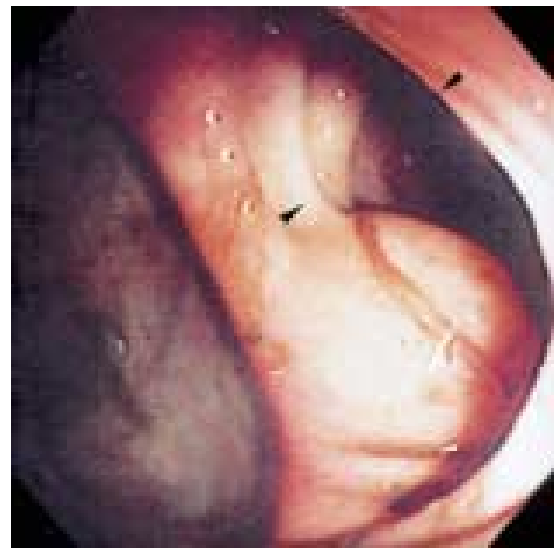

Figure 1 We report a rare case of a patient with a giant diverticulum of the ascending colon who presented with lower gastrointestinal bleeding. Giant diverticulum of the ascending colon is a rare complication of diverticular disease. To our knowledge, this is the first reported case of a wide-base giant diverticulum of the ascending colon complicating lower gastrointestinal bleeding. This is also the first report of the application of colonoscopy in the initial diagnosis of giant colonic diverticulum. The colonoscopic study shows the giant diverticulum with a wide-base opening, measuring $3 \mathrm{~cm}$ in diameter (arrowheads).

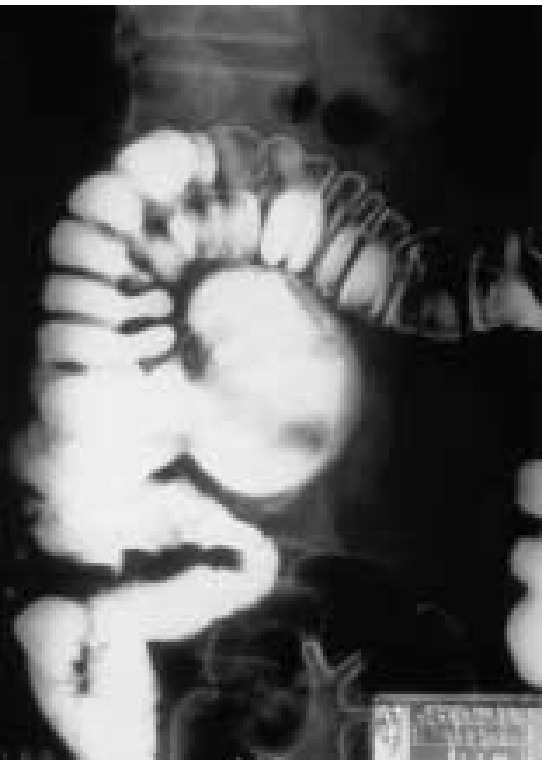

Figure 2 Barium enema examination shows a solitary giant diverticulum of the ascending colon, approximately $9 \times 7 \mathrm{~cm}$ in diameter. 